Internist 2012 $\cdot 53: 249-250$

DOI 10.1007/s00108-011-2937-x

Online publiziert: 26. Februar 2012

(c) Springer-Verlag 2012

M.P. Manns ${ }^{1} \cdot$ S.M. Schellong ${ }^{2} \cdot$ M. Hallek ${ }^{3}$

${ }^{1}$ Klinik für Gastroenterologie, Hepatologie und Endokrinologie, Medizinische Hochschule Hannover

2 II. Medizinische Klinik, Krankenhaus Dresden-Friedrichstadt, Dresden

${ }^{3}$ Klinik I für Innere Medizin - CIO Centrum für Integrierte Onkologie Köln Bonn, Universitätsklinikum Köln

\title{
Sonographie in der Inneren Medizin
}

Es war den Herausgebern eine besondere Freude, das für die praktische Medizin so bedeutsame Schwerpunktheft „Sonographie in der Inneren Medizin“ zusammenzustellen und herauszugeben. In nahezu allen Bereichen der Inneren Medizin ist die Sonographie aufgrund der Echtzeittechnik, der fehlenden Strahlenbelastung und der methodischen Fortschritte, z. B. in der Farbdopplersonographie, Kontrastmittelsonographie und 3-D-Darstellung, von besonderem Wert.

$\mathrm{Zu}$ Beginn werden in einem Grundlagenartikel die methodischen Möglichkeiten dargestellt, darunter auch Verfahren, die noch keinen Einzug in den diagnostischen Alltag gehalten haben, aber an der Schwelle zur klinischen Nutzung im Alltag stehen. Die diagnostische Sonographie ist seit vielen Jahrzehnten ein essenzieller Bestandteil der pränatalen Geburtshilfe. Die abdominelle Sonographie hat längst den Status eines Stethoskops des Internisten und insbesondere des Gastroenterologen erreicht. Ursprünglich war die Domäne der abdominellen Sonographie die Diagnose des Gallensteinleidens. Durch den Einsatz der Kontrastmittelsonographie hat sich jedoch auch die Tumordiagnostik im Abdomen deutlich gebessert. Entscheidend vorangekommen ist ebenso die vaskuläre Diagnostik des Abdomens durch die zunehmende Erfahrung mit der Farbdopplersonographie. Ein Beispiel hierfür ist die Diagnostik des Budd-Chiari-Syndroms. Im Bereich des Abdomens hat sich neben der rein diagnostischen Sonographie inzwischen auch die therapeutische Sonographie für zahlreiche Indika- tionen etabliert. Beispielhaft erwähnt seien die perkutane Sklerosierung von zystischen Raumforderungen der Leber, die sonographisch gesteuerte perkutane Radiofrequenzablation oder Ethanolinjektion von Lebertumoren und auch die sonographisch gesteuerte transkutane Platzierung von Ernährungssonden, so etwa die perkutane Jejunostomie bei voroperiertem Abdomen, wenn eine perkutane Gastrostomie technisch nicht mehr möglich ist. Auch jenseits der Diagnostik und Therapie abdomineller Tumoren ist die Sonographie heute in der Onkologie als Basistechnik anzusehen. Neben der Erkennung von Raumforderungen der Leber, vor allem auch von Lebermetastasen im Rahmen der Tumornachsorge, ist die Erstdiagnostik insbesondere bei Nierentumoren eine Domäne der Sonographie. Gleiches gilt für die Diagnostik von Lymphomen des Bauchraums, aber auch der peripheren Lymphknotenstationen. Bei der Diagnostik nahezu aller subkutanen Tumoren, etwa der Schilddrüse, wurde die Sonographietechnik durch den Einsatz von Kontrastmitteln, Farbdopplersonographie, „daylight imaging“ und 3-dimensionaler Rekonstruktion verfeinert.

Die Nierensonographie ist auch ein wichtiges diagnostisches Verfahren für die Erkennung von parenchymatösen Nierenerkrankungen. Häufig führt die sonographisch gesteuerte Nierenbiopsie zur histologischen Diagnose, beispielsweise nach einer Nierentransplantation oder bei parenchymatösen Nierenerkrankungen. Die Nierensonographie ist auch für die Beurteilung von Steinleiden des Nieren- beckens, der ableitenden Harnwege und der Harnblase von essenzieller Bedeutung. Die farbdopplersonographische Darstellung einer Nierenarterienstenose ist heute der Goldstandard in der Diagnostik des entsprechenden sekundären Hochdruckleidens, das daraufhin umgehend einer Therapie zugeführt werden kann.

\section{》) Heute sind praktisch alle Gefäßprovinzen dem Ultraschall zugänglich}

Schließlich ist die Echokardiographie - ganz gleich, ob sie transthorakal oder transösophageal durchgeführt wird mittlerweile soweit entwickelt, dass Herzvitien, obstruktive oder dilatative Kardiomyopathien, eine systolische oder diastolische Herzinsuffizienz und hypertensive Herzmuskelhypertrophien ohne Hinzuziehung weiterer bildgebender Verfahren einwandfrei diagnostiziert werden können. Der Artikel über die Gefäßsonographie zeigt eindrucksvoll, dass heute praktisch alle Gefäßprovinzen dem Ultraschall zugänglich sind und dass die Duplexsonographie daher zum Standardinstrumentarium der Gefäßmedizin gehört.

Wenngleich die große Bedeutung der Sonographie nicht für alle Bereiche der Inneren Medizin in diesem Schwerpunktheft von Der Internist dargestellt werden kann, hoffen wir doch sehr, Ihnen durch unsere Auswahl einen Einblick zu verschaffen. Perspektivisch zeigt das Beispiel der Gefäßsonographie, welche Möglichkeiten sich hier ergeben werden. Dabei 
wird die Qualität dieser Methodik nicht zuletzt vom Ausbildungsgrad des individuellen Untersuchers abhängig bleiben. Ein akutes Budd-Chiari-Syndrom ist eine lebensbedrohliche Erkrankung. Die oft jungen Patientinnen müssen nach der Diagnose rasch einem spezialisier ten Zentrum zugewiesen werden, in dem eine Lebertransplantation oder die Anlage eines transjugulären intrahepatischen portosystemischen Shunts (TIPSS) im Stadium einer akuten portalen Hypertension möglich ist. Diese therapeutischen Maßnahmen können allerdings nur dann zeitgerecht vollzogen werden, wenn dieses Krankheitsbild in der Initialdiagnostik nicht übersehen wird. Deshalb ist die sonographische Gefäßdiagnostik an jedem Krankenhaus mit Akutversorgung mit ausreichender Kompetenz vorzuhalten. Dies ist nur ein Beispiel dafür, welche Bedeutung die sonographische Ausbildung für Internisten hat. Ihre Bedeutung wird in Zukunft noch zunehmen.

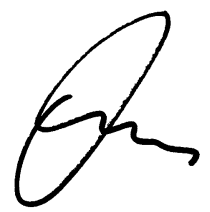

M.P. Manns

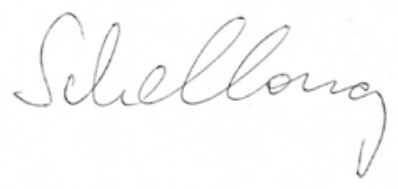

S.M. Schellong

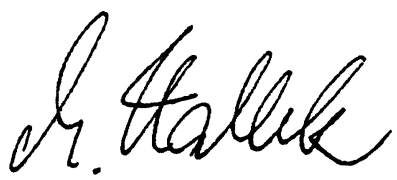

M. Hallek

\section{Korrespondenzadresse}

Prof. Dr. M.P. Manns

Klinik für Gastroenterologie, Hepatologie und Endokrinologie, Medizinische Hochschule Hannover

30625

Carl-Neuberg-Str. 1Hannover

manns.michael@mh-hannover.de

\section{Ausschreibung Oskar Medizin-Preis 2012}

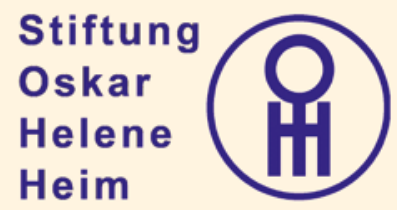

Die Stiftung Oskar-Helene-Heim, deren Zweck die Förderung der Wissenschaft und Forschung ist, verleiht jährlich den mit $\mathbf{5 0 . 0 0 0 €}$ dotierten Oskar Medizin-Preis.

Der Preis wird im Jahr 2012 auf den Gebieten der

\section{Gastroenterologie/Viszeralchirurgie}

ausgeschrieben. Die Therapie von Magenund Darmerkrankungen, die durch das zentrale und enterische Nervensystem verursacht bzw. beeinflusst sind, stellen eine schwerwiegende Problematik dar. Mit dem Preis soll daher ein habilitierter Mediziner ausgezeichnet werden, der zum Thema

\section{„Einfluss des zentralen und enterischen Nervensystems auf Erkrankungen und Funktionen im Magen-Darmbereich"}

besonders relevante Ergebnisse der Grundlagen- und/oder klinischen Forschung in Deutschland erzielt hat. Fachübergreifende Arbeitsgruppen sind ebenfalls zur Bewerbung zugelassen.

Der prämierte Erkenntnisgewinn soll einer breiten Öffentlichkeit vermittelbar sein. Dem Antrag sollen der Lebenslauf, die 5 wichtigsten Arbeiten aus den letzten 3 Jahren zum Thema „Einfluss des zentralen und enterischen Nervensystems auf MagenDarmerkrankungen" sowie eine inhaltliche

Zusammenfassung der Forschungsergebnisse beigefügt werden.

Das Preisgeld ist für Forschungszwecke nach der freien Entscheidung des Preisträgers zu verwenden. Der Preisträger hat der Stiftung diese Verwendung in geeigneter Weise zu belegen. Die Preisverleihung wird im letzten Quartal 2012 in Berlin erfolgen.

Der Antrag ist bis zum 30. Juni 2012 bei der Stiftung Oskar-Helene-Heim einzureichen (www.stiftung-ohh.de).
Weitere Informationen erteilt der Geschäftsführer der Stiftung, Werner Ukas, werner.ukas@stiftung-ohh.de oder info@stiftung-ohh.de, Tel.: 030/8102-1100

Die Schirmherrschaft für diesen Preis hat die Bundesministerin für Bildung und Forschung, Frau Prof. Dr. Annette Schavan, übernommen. Mit diesem Medizinpreis sollen hervorragende Leistungen, insbesondere auf einem der folgenden Gebiete gewürdigt werden: Orthopädie und Orthopädie-Technik, Pneumologie sowie Gastroenterologie/Viszeralchirurgie.

Quelle: Stiftung Oskar-Helene-Heim, www.stiftung-ohh.de 